\title{
Do simultaneous mandibular advancement and temporomandibular joint prosthesis impact the upper airway in TMJ ankylosis patients?
}

\author{
Hui Li ${ }^{1}$, Dongmei He ${ }^{1}$, Qianyang Xie ${ }^{1}$, Ahmed Abdelrehem ${ }^{2}$, Dong Huang ${ }^{1}$, Chi Yang ${ }^{1}$ \\ ${ }^{1}$ Department of Oral Surgery, Shanghai Ninth People's Hospital, College of Stomatology, Shanghai Jiao Tong University School of Medicine, \\ Shanghai Key Laboratory of Stomatology, Shanghai, China; ${ }^{2}$ Department of Craniomaxillofacial and Plastic Surgery, Faculty of Dentistry, Alexandria \\ University, Alexandria, Egypt \\ Contributions: (I) Conception and design: H Li, Q Xie, D He, C Yang; (II) Administrative support: D He, C Yang; (III) Provision of study materials \\ or patients: D He, C Yang; (IV) Collection and assembly of data: H Li, D Huang; (V) Data analysis and interpretation: H Li, Q Xie; (VI) Manuscript \\ writing: All authors; (VII) Final approval of manuscript: All authors. \\ Correspondence to: Dongmei He; Qianyang Xie; Chi Yang. Department of Oral Surgery, Ninth People's Hospital, Shanghai Jiao Tong University \\ School of Medicine, Zhi-Zao-Ju Road, No. 639, Huangpu District, Shanghai 200011, China. Email: lucyhe119@163.com; aqiang2134@126.com; \\ yangchi63@hotmail.com.
}

Background: Ankylosis of the temporomandibular joint (TMJ) is a pathological disorder which results in patients' limited or even complete failure of mouth opening. When TMJ ankylosis occurs during the growing age, moderate to severe micrognathia might be the proposed scenario of such cases, accompanied by obstructive sleep apnea and hypopnea syndrome (OSAHS). TMJ reconstruction using total TMJ prostheses with mandibular advancement (MA) can simultaneously improve the function and aesthetic profile of such patients. The purpose of this study was to determine whether the upper airway can be improved after TMJ reconstruction with total TMJ prostheses combined with or without MA in TMJ ankylosis patients.

Methods: Fourteen patients with pre-(T1) and post-(T2) operative CT scans were included. Patients were divided into two groups according to the operation with or without MA (MA/WoMA). The changes of the upper airway and jaw bones were comparatively analyzed within and between the two groups.

Results: In MA Group, the volume of the total upper airway $(\mathrm{Vt})$ and palatopharynx (V1), together with the surface area (SA) of the total upper airway (SAt) and palatopharynx (SA1) increased significantly after the operation by $41.4 \%, 43.2 \%, 36.3 \%$ and $36.6 \%$, respectively. In WoMA Group, V1, SAt and SA1 increased significantly by $21.0 \%, 19.0 \%$ and $23.1 \%$ following surgery. The changes of Point $\mathrm{B}(\mathrm{P}<0.01)$, Y-axis angle $(\mathrm{P}<0.01)$, SNB $(\mathrm{P}<0.01)$, and ANB $(\mathrm{P}<0.01)$ were significantly greater in MA Group than in WoMA Group. Comparing both groups, the maxilla, and mandible were more backward in MA Group than in WoMA group before the operation, but there was no significant difference of the final position of the maxilla and mandible after the operation between both groups.

Conclusions: Release of TMJ ankylosis and condylar reconstruction using total joint prostheses simultaneously with MA could significantly improve the total volume and other various parameters of the upper airway, while, only the dimension of the palatopharynx increased in cases without MA.

Keywords: Temporomandibular joint (TMJ); ankylosis; TMJ prosthesis; airway remodeling; mandibular advancement (MA)

Submitted Mar 20, 2021. Accepted for publication Oct 12, 2021.

doi: 10.21037/atm-21-1275

View this article at: https://dx.doi.org/10.21037/atm-21-1275 


\section{Introduction}

Temporomandibular joint Ankylosis (TMJA) is a pathological disorder which results in patients' limited or even complete failure of mouth opening. When bilateral TMJA occurs during the growing age, moderate to severe micrognathia might be the proposed scenario of such cases, often accompanied with obstructive sleep apnea and hypopnea syndrome (OSAHS), which can severely influence the patient's quality of life (1). The irreversible damage of the temporomandibular joint (TMJ) is reflected by decreased ramus height, mandibular retrusion and obstructive respiratory disturbances (1). Accordingly, a feasible, reliable and reasonable treatment regimen of ankylosis is therefore crucial and challenging. Based on Yang's classification of TMJ ankylosis (2), TMJA can be classified into two types namely, with or without residual condyle. For those ankyloses with residual condyle, the lateral bony fusion should be eliminated with preservation of the residual condyle. Alternatively, TMJ reconstruction should be chosen for those ankyloses without residual condyle. Compared with the autogenous bone grafting, total TMJ prostheses are more stable without resorption, therefore these can simultaneously improve the function and aesthetic profile of such patients (3).

Our previous study has shown that in cases of TMJ ankylosis without residual condyle, total TMJ prostheses with mandibular advancement (MA) could correct the deformity and dysfunction, with a stable position of jaws and significantly improved mouth opening (4). However, the changes of the upper airway have not been investigated in the previous study. It has been reported that the upper airway enlarged and remained stable in cases of an end-stage TMJ pathology after maxillomandibular counterclockwise rotation $(\mathrm{CCWR})$ and MA with total joint prostheses $(5,6)$. As far as we are concerned, there was no study reporting the evaluation of the upper airway changes in TMJ ankylosis patients undergoing condylar reconstruction with TMJ prosthesis.

The purpose of the current study was to determine whether the upper airway parameters can be improved after TMJ reconstruction with total TMJ prostheses combined with or without MA in TMJ ankylosis patients.

We present the following article in accordance with the STROBE reporting checklist (available at https://dx.doi. org/10.21037/atm-21-1275).

\section{Methods}

This study was approved by the Independent Ethics Committee of Shanghai Jiao Tong University School of Medicine Affiliated 9th People's Hospital (No. SH9H-2014-46) and was conducted in accordance with the Declaration of Helsinki (as revised in 2013). The informed consent was taken from all individual participants.

Patients were recruited based on the following inclusion criteria: (I) all subjects were over 18 years old; (II) patients were diagnosed as unilateral/bilateral skeletal ankylosis of the TMJ without residual condyle; (III) patients underwent unilateral/bilateral TMJ reconstruction with total TMJ prostheses; (IV) CT-scans before and at least 3 months after surgery were taken. The exclusion criteria were as follow: (I) craniofacial syndromes; (II) TMJ reconstruction with autogenous bone grafts (rib grafts or coronoid process grafts, etc.); (III) incomplete clinical or CT data.

All patients were divided into two groups based on whether the MA was performed: (I) TMJ prosthesis with mandible advancement (MA Group); (II) TMJ prosthesis without mandible advancement (WoMA Group). The proper patient selection criteria for mandible advancement in MA group were as follows: (I) patients had a skeletal Class II; (II) SNB less than $73^{\circ}$; (III) patients required to improve the mandibular retrusion deformity.

\section{Technique of operation}

All patients underwent bilateral/unilateral condylar reconstruction with Biomet standard prosthesis (Biomet, Warsaw, Indiana, USA). A modified preauricular incision was applied to expose the bony fusion and upper part of the ramus, combined with a retromandibular incision for exposure of the lower part of the ramus. Digital guides were used to help removing bony fusion, trimming extensive bone spurs in the condylar neck, and previous bone grafting, in addition to guiding a precise prosthesis (Biomet, Warsaw, Indiana, USA) placement.

\section{Combined procedures}

Procedure A, coronoidotomy: the ipsilateral coronoid process was resected simultaneously with the release of the temporalis muscle.

Procedure B, LeFort I osteotomy: the incision was made from first molar to first molar with a $5 \mathrm{~mm}$ of sliding 
Table 1 The definition of relevant landmarks and planes

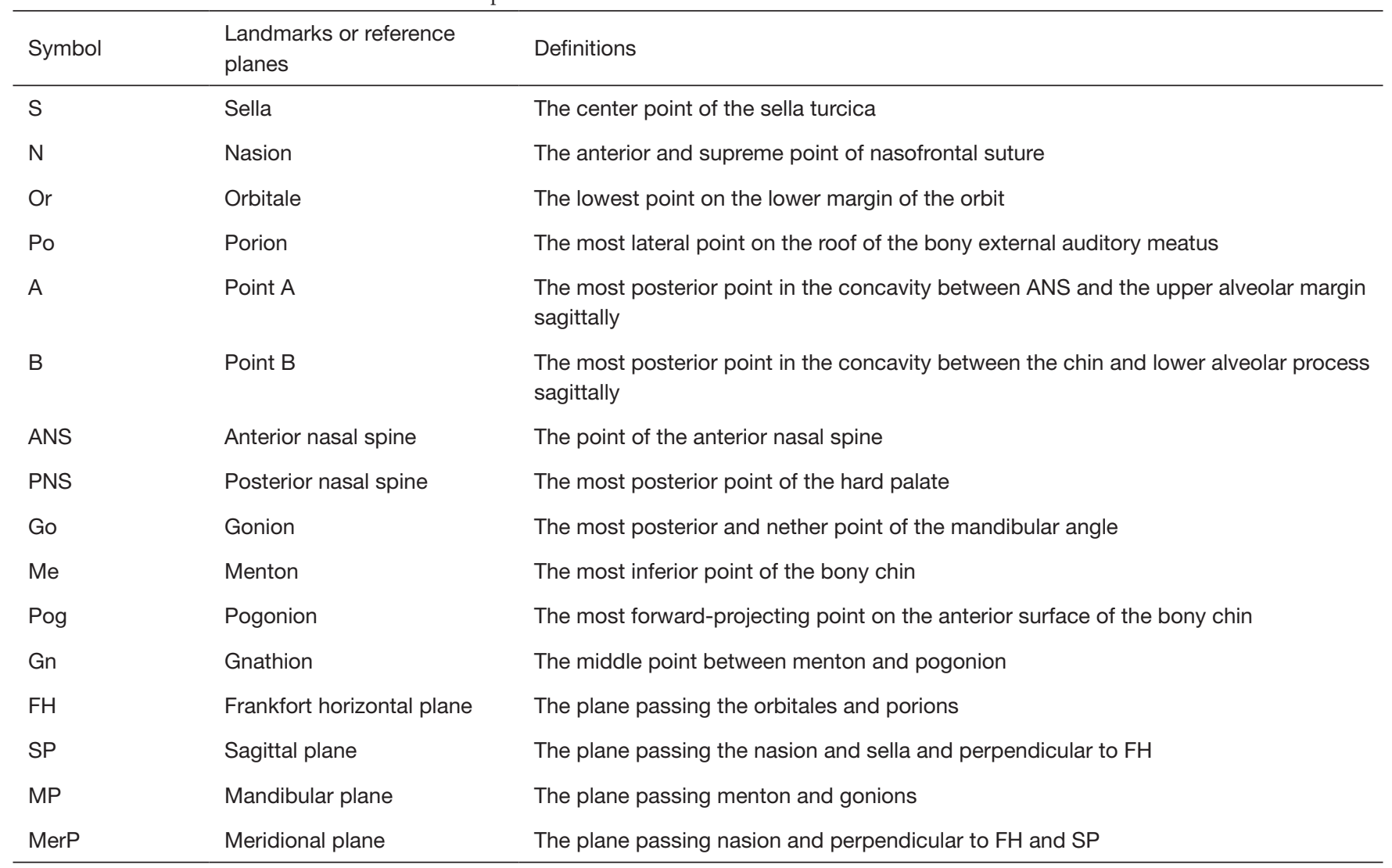

gingival cuff kept on the maxilla. Then, both the lateral and medial buttresses of the maxilla were exposed. Digital guides and surgical splint were applied to help perform the osteotomy and movements of the maxilla and achieve an optimum preoperative plan.

Procedure $C$, sagittal split ramus osteotomy (SSRO): in patients with unilateral TMJ ankylosis, SSRO was performed on the contralateral side when mandibular asymmetry was encountered or mandible advancement was needed. Digital guides and surgical splint were applied to help perform the osteotomy and movements of the mandible.

Procedure D, genioplasty: the incision was made maintaining a $5 \mathrm{~mm}$ of sliding gingival cuff. Medial buttress of the chin was exposed, then the digital guides were applied to help perform the osteotomy and movements of the chin and achieve the preoperative plan.

\section{Measurements}

TMJA patients' spiral maxillofacial CT scans were taken before operation (T1) and at least 3 months after surgery (T2) for all patients. The CT protocol included axial images (matrix size $512 \times 512,120 \mathrm{kVp}$ ) of $1 \mathrm{~mm}$ thickness from the top of the frontal sinuses to the bottom of the mandible. Coronal and sagittal reformats were reconstructed at $0.625 \mathrm{~mm}$ intervals. All the raw CT-scans' data were exported and saved as DICOM format. Imaging Dolphin Version 11.7 (Dolphin Imaging and Management Solution, Chatsworth, Calif., USA) was used to reconstruct the 3-dimensional (3D) upper airway. Proplan CMF 1.4 Software (Materialize, Leuven, Belgium) was used to reconstruct the $3 \mathrm{D}$ maxillofacial hard tissues. All the metrical assessments were performed by the same investigator ( $\mathrm{Li} \mathrm{H}, 13$ years of practice) at $\mathrm{T} 1$ and $\mathrm{T} 2$ and were repeated again after 2 weeks, and then the average value was taken. The details of the computer system performing the analyses were as follows: an operating system, Windows 7 64-bit; Central processing unit, Intel(R) Core(TM) i5-3350P, and Random access memory 4 GB.

The relevant landmarks of the hard tissues were defined and shown in Table 1, and demonstrated in Figure 1. The 

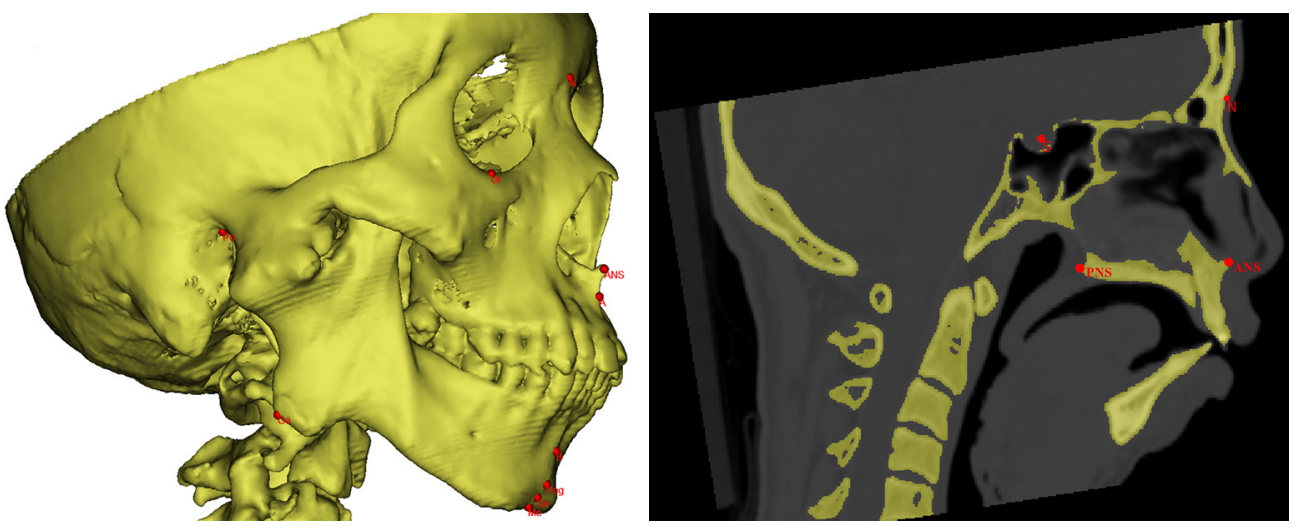

Figure 1 Landmarks of maxilla and mandible. S, sella; N, nasion; Or, orbitale; Po, porion; A, point A; B, point B; ANS, anterior nasal spine; PNS, posterior nasal spine; Go, gonion; Me, menton; Pog, pogonion; Gn, gnathion.

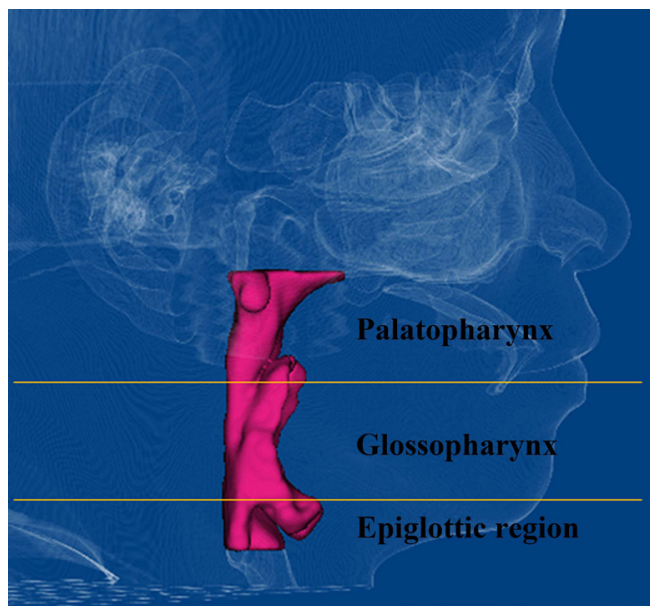

Figure 2 Palatopharynx, from hard palate to the caudal margin of palatine uvula; glossopharynx, caudal margin of palatine uvula to the top of epiglottis; epiglottic region, from the top to the base of epiglottis.

upper airway was divided into three distinct anatomic regions (palatopharynx, glossopharynx, and epiglottic region) (Figure 2), as described in our previous study (7). Frankfort horizontal plane $(\mathrm{FH})$ was taken as a horizontal reference plane, and all other horizontal (transverse) planes of the upper airway measured in this study were parallel to the $\mathrm{FH}$. The anteroposterior plane was adjusted through the ANS and nasion $(\mathrm{N})$ perpendicular to the $\mathrm{FH}$. To evaluate the $3 \mathrm{D}$ and $2 \mathrm{D}$ changes before and after surgery, the volumes, surface area (SA), minimal cross-sectional area (CSA), sagittal diameter (SD), and transverse diameter (TD) of each segmental upper airway were all measured (Table 2, Figure 3).
The measurements of the hard tissue were shown in Table 3. Meridional plane (MerP) was set as being passing through the nasion and perpendicular to the $\mathrm{FH}$ and sagittal plane (SP). In order to evaluate the position of B point, the distance from B to MerP (B-MerP) was measured. If the value of the change of $\mathrm{B}-\mathrm{MerP}$ (T2-T1) was a negative number, this was interpreted that $\mathrm{B}$ point was advanced at $\mathrm{T} 2$, and vice versa. Other measurements of the hard tissue as SNA, SNB, ANB, MP-FH, and Y-axis angle were also measured and compared at $\mathrm{T} 1$ and $\mathrm{T} 2$.

\section{Statistical analysis}

The statistical analyses were carried out using SPSS (Version 16.0; SPSS, Chicago, IL, USA). All measurements were expressed as an average \pm standard deviation. The changes of the upper airway and hard tissue were compared at T1 and T2 within the same group by using the paired $t$ test. An independent sample $t$ test was used for the comparison of the measurements between both groups. Linear regression was applied to analyze the relationship between the changes of Point $\mathrm{B}$ and $\mathrm{Y}$-axis angle and the changes of the measurements of the upper airway. $\mathrm{P}<0.05$ was considered significant.

\section{Results}

Fourteen consecutive patients (10 women and 4 men) were included in this study from March 2014 to February 2018. Nineteen joints (5 patients were bilateral and 9 patients were unilateral) were reconstructed with total joint prosthesis replacement. The average age at surgery was 

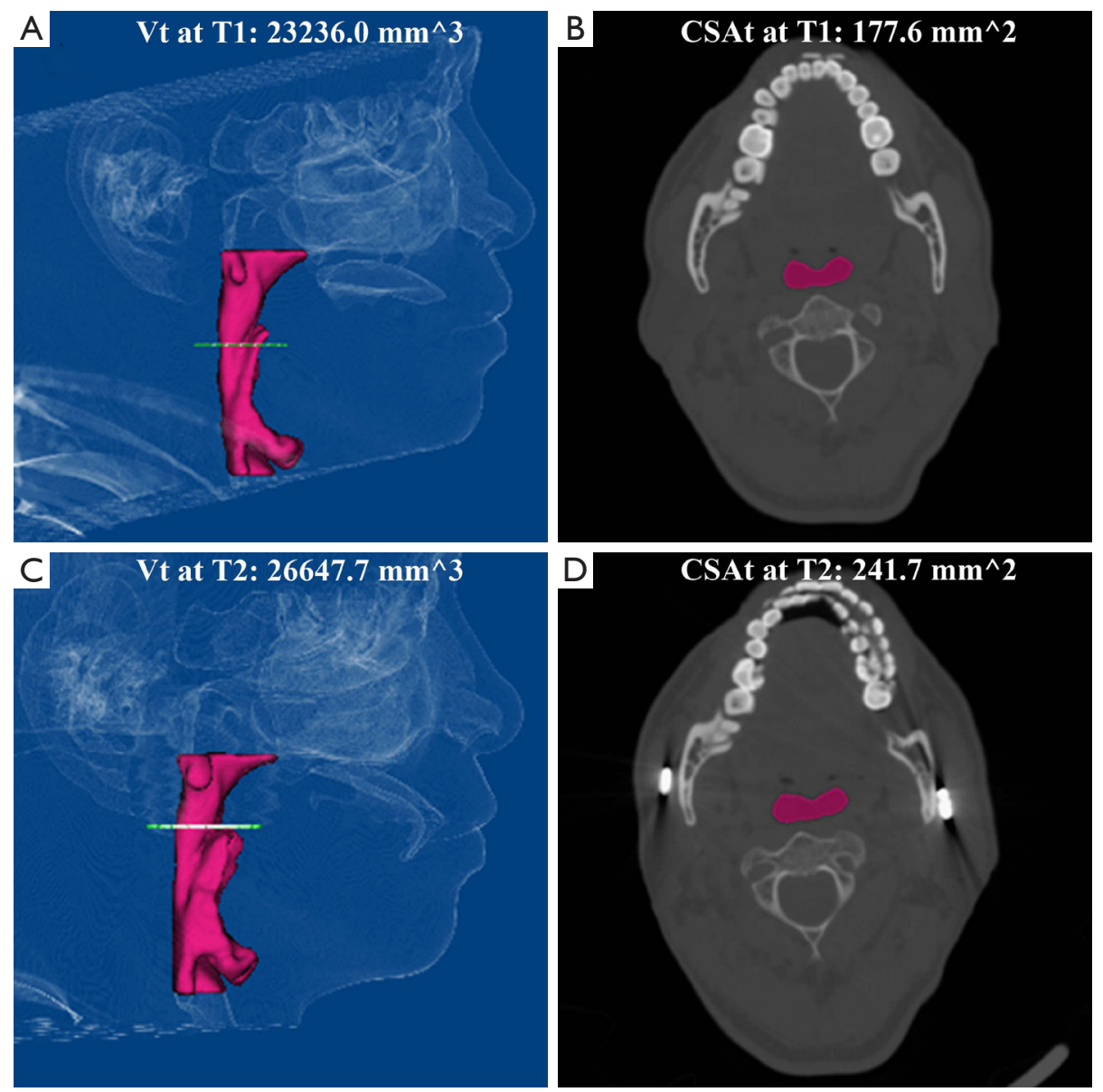

Figure 3 The volume and the minimal cross-sectional area of upper airway were observed before and after operation. (A) The 3-D reconstruction of upper airway before operation; (B) the minimal cross-section of upper airway before operation; (C) the 3-D reconstruction of upper airway after operation; (D) the minimal cross-section of upper airway after operation.

39.4 years (from 20 to 62 years) for the whole sample. The post-operative CT scans were taken at 3 to 27 months after operation (average 7.1 months). There were 7 patients were allocated to MA Group and the other 7 patients to WoMA Group. The average age at surgery was 37.1 years (from 20 to 53 years) in MA Group and 41.6 years (from 25 to 62 years) in WoMA Group. The average age of onset was 7.9 years (from 4 to 13 years) in MA Group and 23.6 years (from 10 to 56 years) in WoMA Group. The average disease course was 29.4 years (from 10 to 49 years) in MA Group and 18 years (from 3 to 40 years) in WoMA Group. There was no significant difference regarding the age at surgery and disease course between the two groups. The age of onset in MA Group was significantly smaller than the age in WoMA Group $(\mathrm{P}<0.05)$.

The detailed information of the included patients in this study was summarized in Table 4.

\section{Maxillofacial hard tissue changes}

The measurements of the maxillofacial hard tissue were shown in the Table 5. The anteroposterior changes of point B (B-MerP, T2-T1) were $9.29 \mathrm{~mm}$ in MA Group $(\mathrm{P}<0.01)$, and $0.26 \mathrm{~mm}$ in WoMA Group $(\mathrm{P}>0.05)$. In MA Group, SNB increased significantly $(\mathrm{P}<0.01)$, while $\mathrm{B}-\mathrm{Mer} \mathrm{P}$ $(\mathrm{P}<0.01)$, ANB $(\mathrm{P}<0.01), \mathrm{MP}-\mathrm{FH}(\mathrm{P}<0.05)$ and $\mathrm{Y}$-axis angle $(\mathrm{P}<0.01)$ decreased at $\mathrm{T} 2$. Comparing both groups, B-MerP $(\mathrm{P}<0.05)$, SNA $(\mathrm{P}<0.01)$ and SNB $(\mathrm{P}<0.05)$ were significantly different at $\mathrm{T} 1$, however without any significant difference at T2. The changes (T2-T1) of B-MerP $(\mathrm{P}<0.01)$, SNB $(\mathrm{P}<0.01)$, ANB $(\mathrm{P}<0.01)$ and $\mathrm{Y}$-axis angle $(\mathrm{P}<0.01)$ were significantly different between both groups. According 
Table 2 The definitions of upper airway parameters

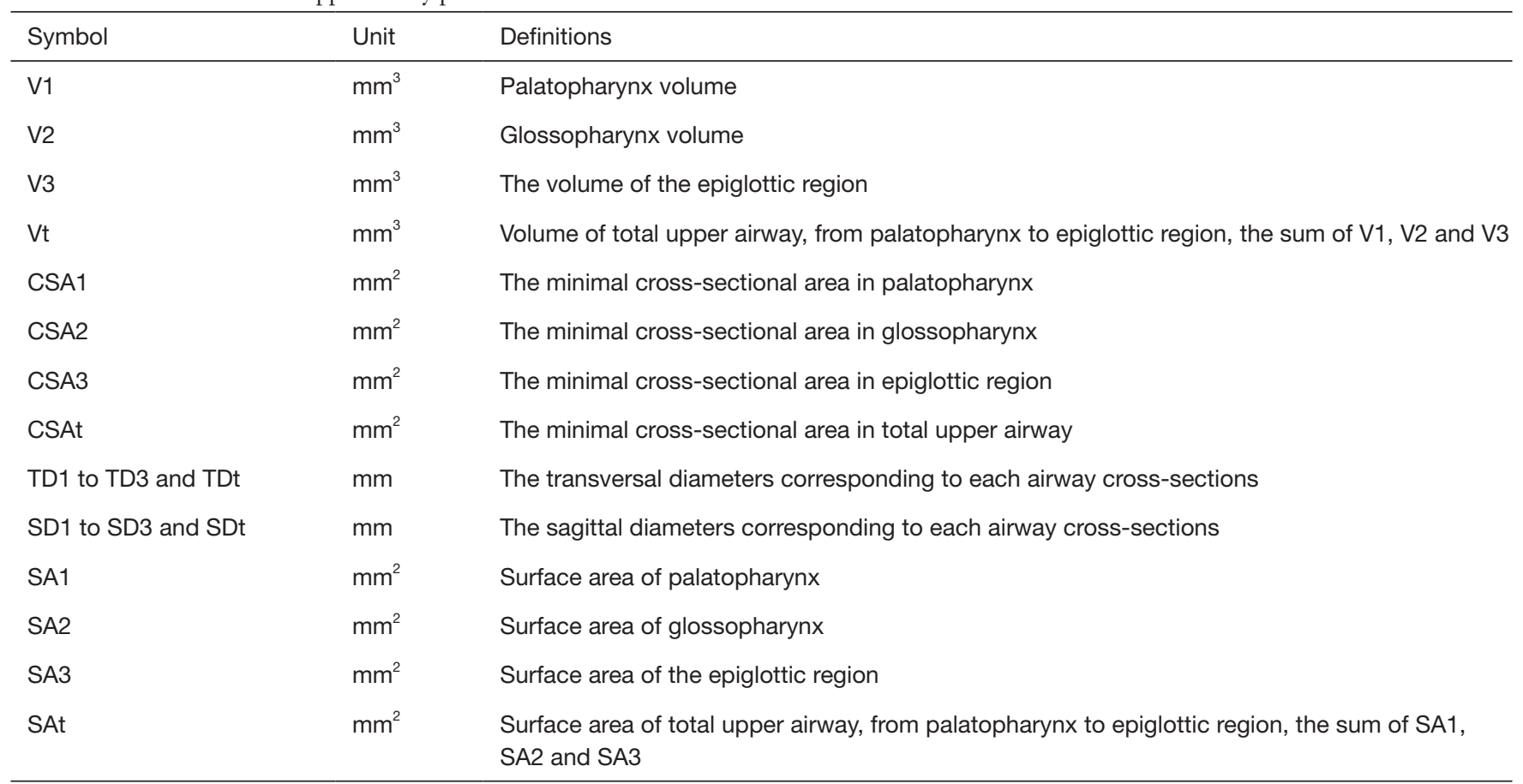

Table 3 The definitions of jaw bone parameters

\begin{tabular}{lll}
\hline Symbol & Unit & Definitions \\
\hline B-MerP & Mm & The distance from B to MerP \\
SNA & Degree & Sella, nasion, and A angle \\
SNB & Degree & Sella, nasion, and B angle \\
ANB & Degree & A, nasion, and B angle \\
MP-FH & Degree & The angle of MP and FH \\
Y-axis angle & Degree & $\begin{array}{l}\text { The angle of FH and the line passing } \\
\text { sella and gnathion }\end{array}$ \\
\hline
\end{tabular}

to the statistical analysis above, the hard tissue changes in MA Group were much greater than in WoMA Group. However, there was no difference of maxillofacial hard tissue changes between bilateral/unilateral side prosthesis in both groups.

\section{Upper airway changes}

The measurements of the upper airway were shown in the Table 6. In MA Group, SAt $(\mathrm{P}<0.01), \mathrm{V} 1(\mathrm{P}<0.05)$, CSAt $(\mathrm{P}<0.01)$, SDt $(\mathrm{P}<0.01)$, SA1 $(\mathrm{P}<0.01), \mathrm{V} 1 \quad(\mathrm{P}<0.01)$, CSA1 $(\mathrm{P}<0.05), \mathrm{SD} 1(\mathrm{P}<0.05)$ and $\mathrm{CSA} 2(\mathrm{P}<0.05)$ increased significantly at T2. The SAt, Vt, SA1 and V1 increased by $36.3 \%, 41.4 \%, 36.6 \%$ and $43.2 \%$ respectively in MA Group.

In WoMA Group, SAt $(\mathrm{P}<0.05)$, SA1 $(\mathrm{P}<0.01)$, V1 $(\mathrm{P}<0.01)$ and $\mathrm{SD} 1(\mathrm{P}<0.01)$ increased significantly at $\mathrm{T} 2$. The SAt, SA1 and V1 increased by $19.0 \%, 23.1 \%$ and $21.0 \%$ respectively in WoMA Group.

There were significant differences in CSAt $(\mathrm{P}<0.05)$ and CSA2 $(\mathrm{P}<0.05)$ at $\mathrm{T} 1$, in addition to the changes (T2-T1) of $\mathrm{V} 1$ between the two groups $(\mathrm{P}<0.05)$. There were no differences of the measurements of the upper airway at $\mathrm{T} 2$.

As shown in Table 7, the results of the linear regression with significance were listed. When the changes (T2-T1) of $\mathrm{B}-\mathrm{MerP}$ and $\mathrm{Y}$-axis angle increased, the changes (T2-T1) of SAt $(\mathrm{P}<0.01), \mathrm{Vt}(\mathrm{P}<0.01), \mathrm{V} 3(\mathrm{P}<0.05)$ and SD2 $(\mathrm{P}<0.05)$ increased consequently.

There were no differences regarding the upper airway changes between bilateral/unilateral side prosthesis in both groups.

\section{Discussion}

An important relationship between maxillofacial development and upper airway has long been investigated in literature. Many patients with an end-stage TMJ pathology 
Table 4 Information of the TMJ ankylosis patients treated by total TMJ prosthesis

\begin{tabular}{|c|c|c|c|c|c|c|c|c|}
\hline No. & Gender & $\begin{array}{c}\text { Age at surgery } \\
\text { (year) }\end{array}$ & $\begin{array}{c}\text { Age of onset } \\
\text { (year) }\end{array}$ & $\begin{array}{c}\text { Course of } \\
\text { disease (year) }\end{array}$ & Group & Sides* & $\begin{array}{c}\text { Combined } \\
\text { Procedures }^{\star \star}\end{array}$ & $\begin{array}{c}\text { Follow up } \\
\text { (month) }\end{array}$ \\
\hline 1 & Male & 41 & 13 & 28 & MA & $\mathrm{Bi}$ & $A+B$ & 7 \\
\hline 3 & Female & 32 & 8 & 24 & MA & $\mathrm{Bi}$ & $A+B+D$ & 5 \\
\hline 6 & Female & 39 & 10 & 29 & WoMA & Uni & D & 8 \\
\hline 7 & Female & 62 & 22 & 40 & WoMA & Uni & - & 3 \\
\hline 8 & Female & 25 & 10 & 15 & WoMA & Uni & $A+B+C+D$ & 5 \\
\hline 12 & Female & 23 & 4 & 20 & MA & Uni & $B+C+D$ & 3 \\
\hline 13 & Female & 27 & 13 & 14 & WoMA & Uni & $B+C+D$ & 3 \\
\hline 14 & Male & 27 & 22 & 5 & WoMA & Uni & A & 3 \\
\hline
\end{tabular}

*: Bi, bilateral; Uni, unilateral; **: A, coronoidotomy; B, LeFort I osteotomy; C, SSRO on the opposite side; D, genioplasty. MA, mandibular advancement; WoMA, without mandibular advancement.

Table 5 The measurements of maxillofacial hard tissue according to the groups and the significance of the comparisons between T1 and T2

\begin{tabular}{|c|c|c|c|c|c|c|c|c|}
\hline Measurements - & \multicolumn{4}{|c|}{ MA } & \multicolumn{4}{|c|}{ WoMA } \\
\hline B-MerP (mm) & $24.18 \pm 9.72^{\circ}$ & $14.89 \pm 8.46$ & $-9.29 \pm 3.89^{\wedge}$ & ** & $12.62 \pm 8.69$ & $12.36 \pm 7.85$ & $-0.26 \pm 2.61$ & - \\
\hline SNA $\left({ }^{\circ}\right)$ & $73.568 \pm 2.79^{\circ \bullet}$ & $75.41 \pm 4.08$ & $1.85 \pm 4.06$ & - & $80.40 \pm 3.40$ & $79.21 \pm 3.30$ & $-1.18 \pm 1.37$ & - \\
\hline SNB $\left({ }^{\circ}\right)$ & $60.87 \pm 5.80^{\circ}$ & $67.36 \pm 5.23$ & $6.49 \pm 4.31^{\Delta \Lambda}$ & ** & $71.11 \pm 8.15$ & $70.76 \pm 7.05$ & $-0.34 \pm 2.50$ & - \\
\hline MP-FH $\left({ }^{\circ}\right)$ & $37.41 \pm 5.78$ & $34.92 \pm 5.89$ & $-2.48 \pm 2.62$ & * & $33.14 \pm 9.81$ & $31.03 \pm 7.32$ & $-2.11 \pm 3.93$ & - \\
\hline Y-axis angle $\left(^{\circ}\right)$ & $72.26 \pm 5.53$ & $68.22 \pm 5.02$ & $-4.04 \pm 1.83^{\mathbf{\Lambda}}$ & ** & $66.03 \pm 5.36$ & $65.83 \pm 5.20$ & $-0.20 \pm 1.43$ & - \\
\hline
\end{tabular}

T2 compared with $\mathrm{T} 1$ in group: ${ }^{*}, \mathrm{P}<0.05 ;{ }^{* *}, \mathrm{P}<0.01$. T1 compared between groups: ${ }^{\bullet}, \mathrm{P}<0.05 ;{ }^{\circ}, \mathrm{P}<0.01$. T2-T1 compared between groups: ${ }^{\boldsymbol{\Lambda}}, \mathrm{P}<0.01$. MA, mandibular advancement; WoMA, without mandibular advancement.

present with common features including high occlusal plane and mandible retrusion in a downward and backward way (8). Such maxillofacial features may be related to a decreased oropharyngeal airway (5) and respiratory disturbances due to an upper airway obstruction (1).

TMJ ankylosis without residual condyle is a type of an irreversible TMJ damage (2). For such patients, it might be necessary to reconstruct the TMJ and simultaneously advance the mandible (9). Previous reports have documented the effectiveness of MA in patients with an end-stage TMJ pathology $(3,6)$.

In the present study, 3-D linear, angular and volumetric parameters were measured to indicate the changes of the upper airway and jaws. Lateral cephalograms have traditionally been applied to evaluate the upper airway parameters, however, only providing 2-D images. CT, 


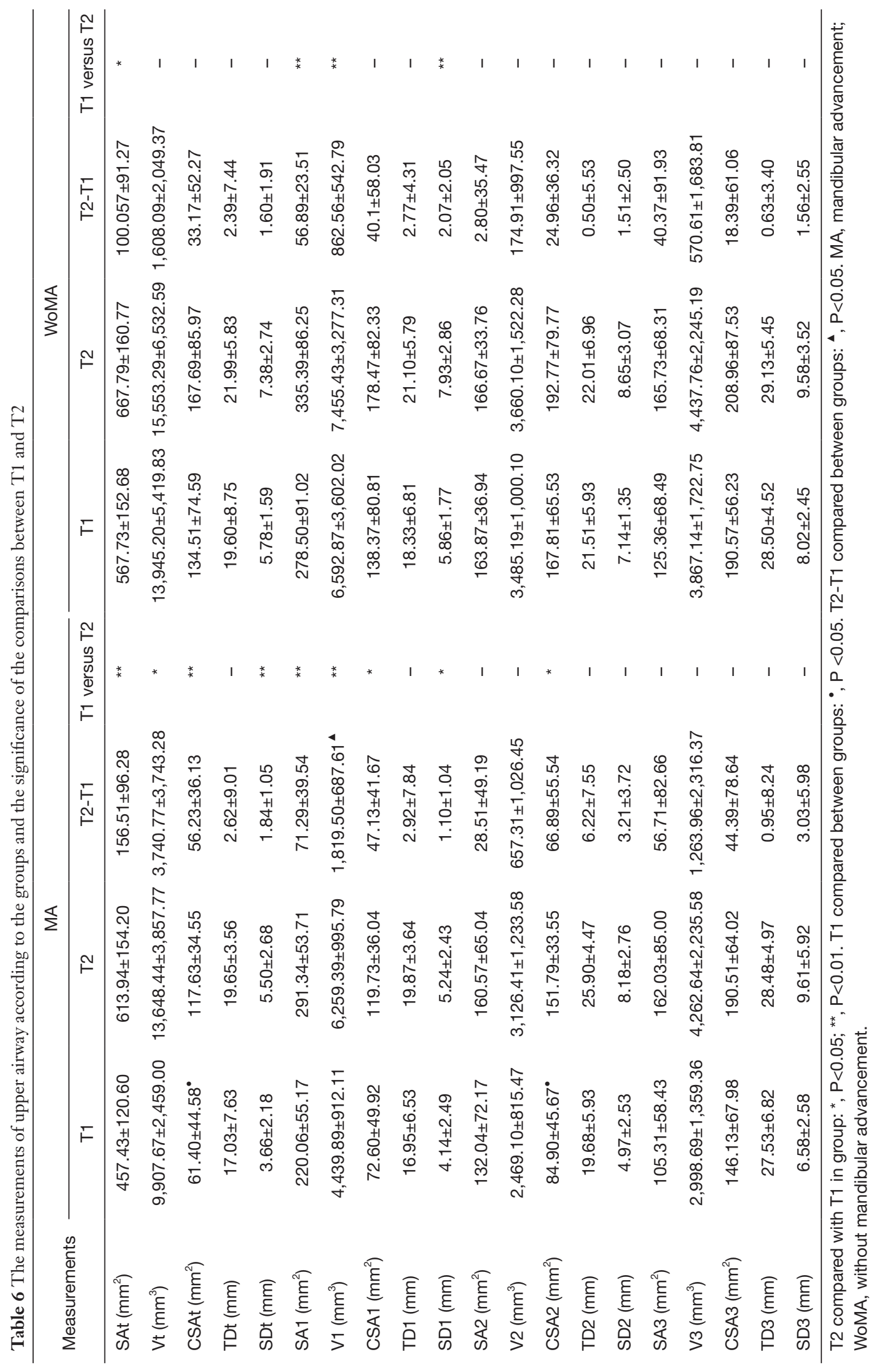


Table 7 The results of linear regression with significance

\begin{tabular}{|c|c|c|}
\hline $\mathrm{T} 2-\mathrm{T} 1$ & B-MerP (T2-T1) & Y-axis angle (T2-T1) \\
\hline SAt & $0.005^{\star \star}$ & $0.009^{\star \star}$ \\
\hline SA3 & $0.048^{*}$ & - \\
\hline Vt & $0.009^{\star \star}$ & $0.002^{\star \star}$ \\
\hline V1 & $0.013^{\star}$ & - \\
\hline V3 & $0.024^{*}$ & $0.029^{*}$ \\
\hline SD1 & - & $0.033^{*}$ \\
\hline SD2 & $0.017^{\star}$ & $0.030^{*}$ \\
\hline SD3 & $0.046^{*}$ & - \\
\hline
\end{tabular}

*, $\mathrm{P}<0.05 ;{ }^{* *}, \mathrm{P}<0.01$.

which can provide more useful quantitative and qualitative data, has long been considered a reliable alternative tool to assess the upper airway (10).

In MA Group, the changes of point B were statistically significant $(\mathrm{P}<0.01)$ following surgery with an average of $9.29 \mathrm{~mm}$ (from 5.2 to $15.08 \mathrm{~mm}$ ). In Wolford et al.'s study (3) and Gonçalves et al.'s study (6), the average anteroposterior changes of point $B$ were $13.4 \mathrm{~mm}$ (from 4 to $25 \mathrm{~mm}$ ), and 9.93 (from 8.37 to $11.5 \mathrm{~mm}$ ), respectively. Zinser et al.'s investigated patients with OSAHS who underwent MA and CCWR (11), reporting that the anteroposterior changes of the mandible were from 9 to $15 \mathrm{~mm}$, with an average of $11.84 \mathrm{~mm}$.

Before surgery, SNA and SNB in MA Group were significantly smaller and also Point $\mathrm{B}$ was significantly more backward than those in WoMA Group, indicating that the patients in MA Group had more backward jaws. Moreover, the age of onset in MA Group (7.9 years) was significantly smaller than that in WoMA Group (23.6 years), implying that micrognathia might occur during the growing age. After surgery, there was no significant difference of maxillofacial hard tissues between both groups. As a result, the changes of Point B, Y-axis angle, SNB and ANB in MA Group were significantly greater than those in WoMA Group. This indicated that the final postoperative profiles of patients in both groups were similar.

In this study, the changes of the upper airway in MA Group were significant. In WoMA Group, the palatopharynx was the only individual segment of the upper airway that had significant changes, which indicated that the palatopharynx (SA1, V1 and SD1) could be expanded whether with MA or not, however, V1 could increase much greater after MA. It was an interesting finding that releasing ankylosis combined with TMJ reconstruction alone (without MA) could achieve an enlarged palatopharynx. Such results were in accordance with the results of Liu et al. (12) revealing an increased SD of the palatopharynx following condylar reconstruction with an autogenous coronoid process graft without MA.

The minimum CSA is an important index for the airway function, which is associated with the occurrence of OSAHS (10). The minimum CSA of the total upper airway, palatopharynx and glossopharynx at T2 was significantly larger than the CSA at T1 in MA Group. Considering the statistical analysis of all patients, the anteroposterior changes of point $\mathrm{B}$ and $\mathrm{Y}$-axis angle were significantly related to the changes of the dimension of the total and segmental upper airways, that in turn documented the effectiveness of MA in another way.

However, from the results of the current study, it can be demonstrated that there was no difference of the upper airway or hard tissue changes in patients that had bilateral total joint replacement versus unilateral total joint replacement. The limited number of cases might be one of the reasons for such finding. But it has been implied that the changes of the upper airway might be more influenced by various surgical procedures. MA is considered the most important surgical procedure to increase the dimension of the upper airway according to the results presented in our study. Other combined procedures had their own different goals. Coronoidotomy aimed to release the extraarticular tension, while LeFort I osteotomy and SSRO were planned when correction of facial asymmetry or MA were needed. Genioplasty would be performed for further correction of mandibular retrusion. The outcomes obtained from this study were in an agreement with other studies $(6,10,12,13)$, revealing a significant increase in the upper airway volume following various surgical procedures. Bi et al. (13) showed that the CSA and the three segmentations of the upper airway volume were significantly increased following distraction osteogenesis (DO) in TMJ ankylosis patients. However, the changes of point $\mathrm{B}$ were not investigated nor analyzed (13). Liu et al. (12) reported a significantly increased upper airway volume at 2 weeks after TMJ reconstruction with coronoid process in TMJ ankylosis patients, but slightly decreased later at 13 months postoperatively. Still the changes of point B were not also investigated. In Costa et al.'s study (14), it was revealed that the mandibular rotation (open- and closed-mouth positions) alone could change the volume of upper airway. 
Hence, MA and CCWR were often used as combined procedures in many studies $(5,6,10)$. Gonçalves et al. showed a significant increase in the volume and CSA of the upper airway immediately after simultaneous MA and CCWR with TMJ prostheses (6). According to Louro et al.'s review (10), the procedure of MA with CCWR revealed a significant increase in the volume of the upper airway space. MA affects the walls of the pharynx by "tightening" the pharyngeal airway musculature (15), and this offers a stable and significant reduction in the collapsibility of the hypopharynx (11).

There was no significant change neither in the MA group nor in the WoMA group about the epiglottic region, however, the V3 (T2-T1) and SD3 (T2-T1) significantly increased with MA. These results about the epiglottic region were in agreement with Kim et al.'s study (16). In Zinser et al.'s study (11), the volume, length, CSA and SD of the epiglottic region increased significantly after MA and CCWR. As mentioned before, the average change of MA was greater in Zinser et al.'s study (11.84 mm) (11) than in our study $(9.29 \mathrm{~mm})$. And this might explain why the change of the epiglottic region was insignificant in the current study.

Autogenous bone grafts (rib grafts and coronoid process grafts) and Total TMJ prosthesis have long been used for reconstructing the TMJ. Autogenous bone grafts have unpredictable and unsatisfactory results such as ankylosis recurrence and growth disturbances $(17,18)$. In our previous study of TMJ ankylosis, reconstruction with rib grafts in the growing children could achieve good mouth opening, however, an asymmetric growth (1 in 7 patients) and reankylosis ( 1 in 7 patients) were observed after an average of 46.4 months' follow-up (18). Moreover, bone resorption of rib grafts and coronoid process grafts was also reported (19). Moreover, it was advocated that the height of mandibular ramus decreased in long-term-follow-up when the coronoid process graft was used in TMJ reconstruction (12). In comparison, TMJ reconstruction with total joint prostheses remained stable over the follow-up period considering the hard tissue parameters (6). Total joint prosthesis is a reliable method for TMJ reconstruction (4), and it provides better stability and improves the function and esthetics (5). Moreover, according to this study, the dimensions of the upper airway were significantly improved, added to the above outcomes.

Still this study has some limitations. First, the average follow up period after total TMJ prostheses replacement was only 7.1 months. Therefore, longer-term follow up periods would be needed to evaluate the changes of the airway dimensions and stability of the hard tissue. Second, the number of the included cases was limited. Hence, a greater number of patients would be necessary to investigate further difference between genders or bilateral/ unilateral side prosthesis. Third, the data of patients' height and weight were not collected. The correlations between the changes of the airway dimensions and height, weight or body mass index (BMI) could not be examined in this study. Fourth, according to the records, there were some patients with complains of sleeping apnea or snoring. But they did not undergo polysomnography before or after surgery, and it was not available whether breathing or obstructive sleep apnea had been improved. Accordingly, further prospective studies, with well-designed research methods and more detailed data would find more reliable and outstanding outcomes.

\section{Conclusions}

In conclusion, release of TMJ ankylosis and condylar reconstruction using total joint prostheses simultaneously with MA could significantly improve the total volume and other parameters of the upper airway, while, only the dimension of the palatopharynx increased in cases without MA.

\section{Acknowledgments}

We would like to thank Huda Abdelrehem for her help in polishing our paper.

Funding: This work was supported by the Interdisciplinary Program of Shanghai Jiao Tong University [YG2019QNB08], the Science and Technology Commission of Shanghai Municipality Science Research Project [20Y11903900, 20S31902500], and the Clinical plus project of Shanghai 9th People's Hospital [JYLJ201805].

\section{Footnote}

Reporting Checklist: The authors have completed the STROBE reporting checklist. Available at https://dx.doi. org/10.21037/atm-21-1275

Data Sharing Statement: Available at https://dx.doi. org/10.21037/atm-21-1275

Peer Review File: Available at https://dx.doi.org/10.21037/ 
atm-21-1275

Conflicts of Interest: All authors have completed the ICMJE uniform disclosure form (available at https://dx.doi. org/10.21037/atm-21-1275). The authors have no conflicts of interest to declare.

Ethical Statement: The authors are accountable for all aspects of the work in ensuring that questions related to the accuracy or integrity of any part of the work are appropriately investigated and resolved. The study was conducted in accordance with the Declaration of Helsinki (as revised in 2013). The study was approved by the Independent Ethics Committee of Shanghai Jiao Tong University School of Medicine Affiliated 9th People's Hospital (No. SH9H-2014-46) and informed consent was taken from all individual participants.

Open Access Statement: This is an Open Access article distributed in accordance with the Creative Commons Attribution-NonCommercial-NoDerivs 4.0 International License (CC BY-NC-ND 4.0), which permits the noncommercial replication and distribution of the article with the strict proviso that no changes or edits are made and the original work is properly cited (including links to both the formal publication through the relevant DOI and the license). See: https://creativecommons.org/licenses/by-nc-nd/4.0/.

\section{References}

1. Mishima K, Yamada T, Sugahara T. Evaluation of respiratory status and mandibular movement after total temporomandibular joint replacement in patients with rheumatoid arthritis. Int J Oral Maxillofac Surg 2003;32:275-9.

2. He D, Yang C, Chen M, et al. Traumatic temporomandibular joint ankylosis: our classification and treatment experience. J Oral Maxillofac Surg 2011;69:1600-7.

3. Wolford LM, Pinto LP, Cárdenas LE, et al. Outcomes of treatment with custom-made temporomandibular joint total joint prostheses and maxillomandibular counter-clockwise rotation. Proc (Bayl Univ Med Cent) 2008;21:18-24.

4. Hu Y, Zhang L, He D, et al. Simultaneous treatment of temporomandibular joint ankylosis with severe mandibular deficiency by standard TMJ prosthesis. Sci Rep 2017;7:45271.
5. Coleta KE, Wolford LM, Gonçalves JR, et al. Maxillomandibular counter-clockwise rotation and mandibular advancement with TMJ Concepts total joint prostheses: part II--airway changes and stability. Int J Oral Maxillofac Surg 2009;38:228-35.

6. Gonçalves JR, Gomes LC, Vianna AP, et al. Airway space changes after maxillomandibular counterclockwise rotation and mandibular advancement with TMJ Concepts ${ }^{\circledR}$ total joint prostheses: three-dimensional assessment. Int J Oral Maxillofac Surg 2013;42:1014-22.

7. Li H, Lu X, Shi J, et al. Measurements of normal upper airway assessed by 3-dimensional computed tomography in Chinese children and adolescents. Int J Pediatr Otorhinolaryngol 2011;75:1240-6.

8. Oulis CJ, Vadiakas GP, Ekonomides J, et al. The effect of hypertrophic adenoids and tonsils on the development of posterior crossbite and oral habits. J Clin Pediatr Dent 1994;18:197-201.

9. Wolford LM, Cottrell DA, Henry CH. Temporomandibular joint reconstruction of the complex patient with the Techmedica custom-made total joint prosthesis. J Oral Maxillofac Surg 1994;52:2-10; discussion 11.

10. Louro RS, Calasans-Maia JA, Mattos CT, et al. Three-dimensional changes to the upper airway after maxillomandibular advancement with counterclockwise rotation: a systematic review and meta-analysis. Int J Oral Maxillofac Surg 2018;47:622-9.

11. Zinser MJ, Zachow S, Sailer HF. Bimaxillary 'rotation advancement' procedures in patients with obstructive sleep apnea: a 3-dimensional airway analysis of morphological changes. Int J Oral Maxillofac Surg 2013;42:569-78.

12. Liu L, Li J, Ji H, et al. Cone-beam computed tomography evaluation of the maxillofacial features of patients with unilateral temporomandibular joint ankylosis undergoing condylar reconstruction with an autogenous coronoid process graft. PLoS One 2017;12:e173142.

13. Bi RY, Luo XT, Jiang N, et al. Change in the posterior airway after mandibular distraction osteogenesis in patients with ankylosis of the temporomandibular joint: a retrospective study. Br J Oral Maxillofac Surg 2018;56:525-30.

14. Costa ALF, Castilho Fardim KA, de Almeida BM, et al. Eccentric movements of temporomandibular joint and upper airway volume: Three-dimensional segmentation using cone beam computed tomography. Cranio 2021. [Epub ahead of print].

15. Li KK, Guilleminault C, Riley RW, et al. Obstructive 
sleep apnea and maxillomandibular advancement: an assessment of airway changes using radiographic and nasopharyngoscopic examinations. J Oral Maxillofac Surg 2002;60:526-30; discussion 531.

16. Kim JS, Kim JK, Hong SC, et al. Changes in the upper airway after counterclockwise maxillomandibular advancement in young Korean women with class II malocclusion deformity. J Oral Maxillofac Surg 2013;71:1603.e1-6.

17. Gupta GM, Gupta P, Sharma A, et al. Evaluation of functional and esthetic outcome after correction of mandibular hypoplasia secondary to temporomandibular

Cite this article as: Li H, He D, Xie Q, Abdelrehem A, Huang D, Yang C. Do simultaneous mandibular advancement and temporomandibular joint prosthesis impact the upper airway in TMJ ankylosis patients? Ann Transl Med 2021;9(22):1638. doi: 10.21037/atm-21-1275 ankylosis treated by distraction osteogenesis. J Maxillofac Oral Surg 2014;13:152-8.

18. Zhao J, He D, Yang C, et al. 3-D computed tomography measurement of mandibular growth after costochondral grafting in growing children with temporomandibular joint ankylosis and jaw deformity. Oral Surg Oral Med Oral Pathol Oral Radiol 2017;124:333-8.

19. Huang D, Lu C, Yao Z, et al. A Comparison of the Effect Between Coronoid Process Graft and Costochondral Graft in the Reconstruction of Temporomandibular Joint. J Craniofac Surg 2016;27:e197-200. 